\title{
Netrin-1 Acts as a Repulsive Guidance Cue for Sensory Axonal Projections toward the Spinal Cord
}

\author{
Tomoyuki Masuda, ${ }^{1 \star}$ Keisuke Watanabe, ${ }^{2,3 *}$ Chie Sakuma, ${ }^{1 *}$ Kazuhiro Ikenaka, ${ }^{3}$ Katsuhiko Ono, ${ }^{3}$ and \\ Hiroyuki Yaginuma ${ }^{1}$ \\ ${ }^{1}$ Department of Anatomy, Fukushima Medical University School of Medicine, Fukushima 960-1295, Japan, 2Department of Morphological Neural Science, \\ Graduate School of Medical Sciences, Kumamoto University, Kumamoto 860-8556, Japan, and ${ }^{3}$ Division of Neurobiology and Bioinformatics, National \\ Institute for Physiological Sciences, Okazaki 444-8787, Japan
}

During early development, the ventral spinal cord expresses chemorepulsive signals that act on dorsal root ganglion (DRG) axons to help orient them toward the dorsolateral part of the spinal cord. However, the molecular nature of this chemorepulsion is mostly unknown. We report here that netrin-1 acts as an early ventral spinal cord-derived chemorepellent for DRG axons. In the developing mouse spinal cord, netrin-1 is expressed in the floor plate of the spinal cord, and the netrin receptor Unc $5 c$ is expressed in DRG neurons. We show that human embryonic kidney cell aggregates secreting netrin-1 repel DRG axons and that netrin-1-deficient ventral spinal cord explants lose their repulsive influence on DRG axons. In embryonic day 10 netrin-1 mutant mice, we find that DRG axons exhibit transient misorientation. Furthermore, by means of gain-of-function analyses, we show that ectopic netrin-1 in the dorsal and intermediate spinal cord prevents DRG axons from being directed toward the dorsal spinal cord. Together, these findings suggest that netrin-1 contributes to the formation of the initial trajectories of developing DRG axons as a repulsive guidance cue.

Key words: spinal cord; dorsal root ganglion; chemorepulsion; netrin-1; axonal guidance; Unc5; floor plate

\section{Introduction}

The formation of functional neuronal circuits depends on the appropriate projection of axons to their targets. During their elongation, axons are guided to their targets by attractive and repulsive cues working in a contact-dependent or diffusible manner (for review, see Tessier-Lavigne and Goodman, 1996). During the initial stage of dorsal root ganglion (DRG) axonal growth, surrounding "nontarget" tissues such as the dermamyotome, the notochord and the ventral spinal cord release strong chemorepulsive signals for DRG axons (Keynes et al., 1997; Nakamoto and Shiga, 1998). We reported previously that semaphorin $3 \mathrm{~A}$ (sema3A) participates in the chemorepulsive activities from the notochord and the dermamyotome (supplemental Fig. 1, available at www.jneurosci.org as supplemental material) (Masuda et al., 2003). However, the molecular nature of early ventral spinal cord-derived chemorepulsion is less clear, because the initial ax-

Received Dec. 5, 2007; revised July 14, 2008; accepted Sept. 9, 2008.

This work was supported by grants from the program Grants-in-Aid for Scientific Research of the Ministry of Education, Culture, Sports, Science, and Technology, Japan, by the Cooperative Study Program of the National Institute for Physiological Sciences, and by grants from the Brain Science Foundation, Japan. Dr. M. Tessier-Lavigne kindly provided netrin-1-deficient mice and pCAG-chick netrin-1. We thank Drs. F. Murakami, N. Tamamaki, and H. Takebayashi for the gift of plasmids, and K. Inaba for assistance.

*T.M., K.W., and C.S. contributed equally to this work.

Correspondence should be addressed to Dr. Tomoyuki Masuda, Fukushima Medical University School of Medicine, 1 Hikarigaoka, Fukushima City, Fukushima 960-1295, Japan. E-mail: tmasu@fmu.ac.jp.

K. Ono's present address: Department of Biology, Kyoto Prefectural University of Medicine, Kyoto 603-8334, Japan.

D0I:10.1523/JNEUROSCI.1926-08.2008

Copyright $\odot 2008$ Society for Neuroscience ～0270-6474/08/2810380-06\$15.00/0 onal projections from DRG neurons appear to be normal in Sema3A mutants (Taniguchi et al., 1997).

Netrin-1 is a bifunctional axonal guidance molecule (Kennedy et al., 1994; Serafini et al., 1994; Colamarino and Tessier-Lavigne, 1995). Floor plate-derived netrin-1 regulates circumferential axonal projections, acting as a chemoattractive or chemorepulsive cue depending on the specific combination of deleted in colorectal cancer (DCC) and Unc5 receptors (Hong et al., 1999). Netrin-1 is also transiently expressed in the developing dorsal spinal cord, which inhibits DRG axonal growth and prevents the premature extension of DRG axons into the mantle layer at later stages via the Unc5 receptor (Watanabe et al., 2006). Thus, netrin-1 provides an inhibitory cue for DRG axonal guidance in the early spinal cord, but it is not clear whether DRG axons respond to ventrally derived netrin-1 before reaching the spinal cord.

In the present study, we examined the role of netrin- 1 in the formation of the initial DRG projections. We found that early ventral spinal cord-derived netrin-1 participates in DRG axonal guidance via its chemorepulsive action both in vivo and in vitro.

\section{Materials and Methods}

Animals. Fertilized chicken eggs were incubated at $37.6^{\circ} \mathrm{C}$ until they reached the appropriate age (stage 16-24 of Hamburger and Hamilton, 1992). Mice homozygous for netrin-1 mutations were obtained from heterozygote matings and identified as described previously (Serafini et al., 1996). Experiments using netrin-1 mutant mice were approved by the Animal Research Committee of the National Institute for Physiological Sciences. Embryonic day 0.5 (E0.5) was defined as the midday of vaginal plug discovery. All analyses in this study were performed on the thoracic spinal cord. 


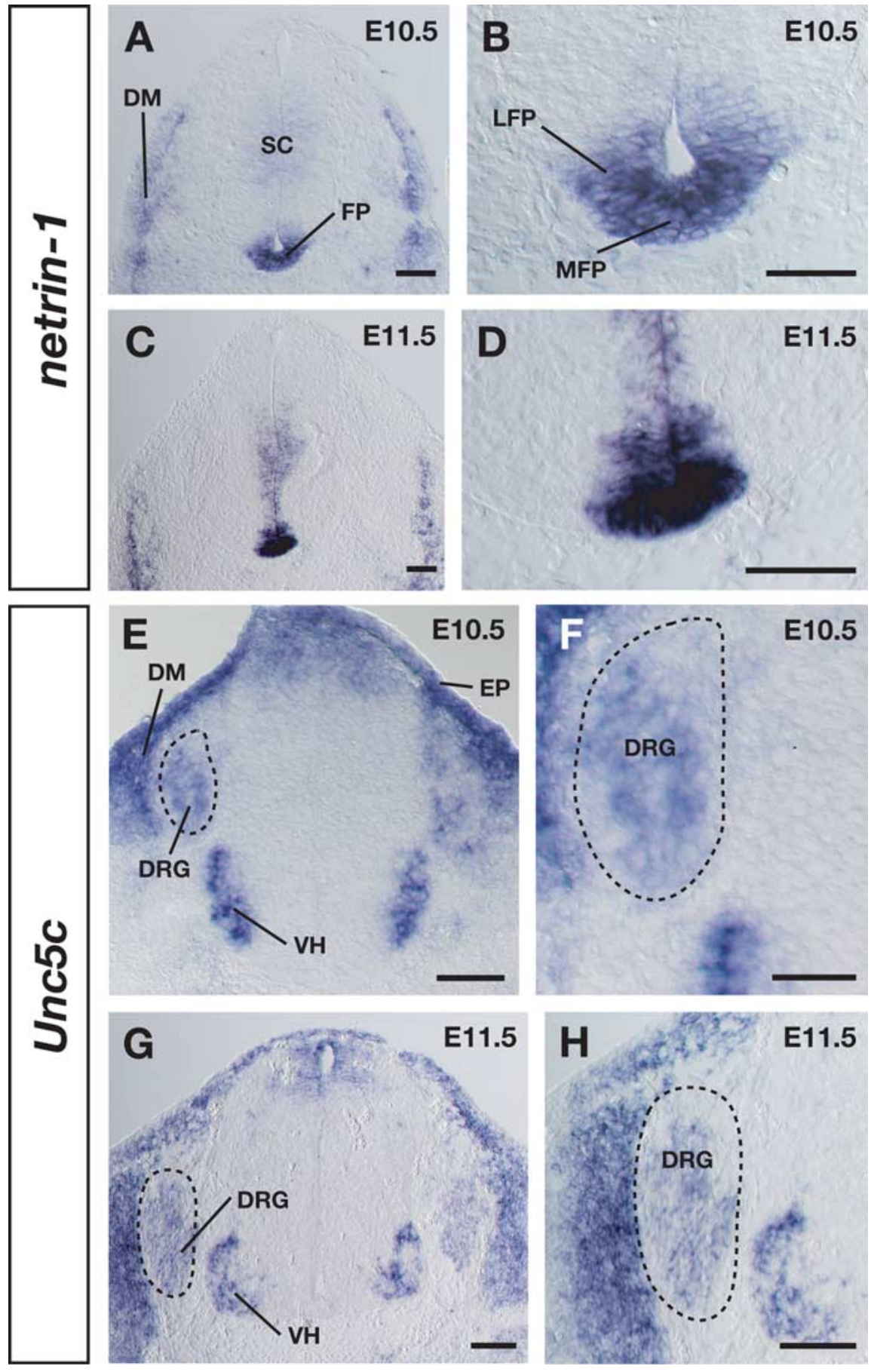

Figure 1. Expression of netrin- 1 and its receptor Unc 5 c in the mouse embryo. $A, A t E 10.5$, netrin- 1 is expressed in the floor plate (FP) and dermamyotome (DM). B, A higher-magnification view of the floor plate in $A$. $C$, At E11.5, netrin-1 expression in the floor plate continued. D, A higher-magnification view in $\boldsymbol{C}$. $\boldsymbol{E}$, At E10.5, the netrin receptor Unc 5 c is expressed in the DRG neurons (dashed circle), dermamyotome, epidermis (EP), and ventral horn (VH). $\boldsymbol{F}$, A higher-magnification view of the DRG region in $\boldsymbol{E}$. $\boldsymbol{G}$ At E11.5, Unc5c expression continued in DRG neurons (dashed circle). $\boldsymbol{H}$, A higher-magnification view of $\boldsymbol{G}$. SC, Spinal cord; LFP, lateral floor plate; MFP, medial floor plate. Scale bars: $A-E, G, H, 100 \mu \mathrm{m} ; \boldsymbol{F}, 50 \mu \mathrm{m}$.

In situ hybridization. In situ hybridization was performed as described previously (Ding et al., 2005), using the following digoxygenin (DIG)labeled RNA probes: mouse netrin-1, Unc5c (formerly Unc5h3), DCC, and chick netrin-1 (Sugimoto et al., 2001; Watanabe et al., 2006). DIG-labeled RNA probes were synthesized by using a DIG RNA-labeling kit (Roche Diagnostics).

Immunohistochemistry and analysis of DRG axonal projection. For staining DRG axons in vivo, E10-E17.5 mouse or stage 22 chick embryos were fixed with $4 \%$ paraformaldehyde (PFA) overnight. After immersion in graded sucrose solutions, tissues were frozen in Tissue-Tek OCT compound (Bayer), and transverse sections $(18 \mu \mathrm{m})$ were cut with a cryostat and collected onto MAS-coated glass slides (Matsunami). The anti-class III $\beta$-tubulin $(1 \mu \mathrm{g} / \mathrm{ml}$; Promega) for the detection of neurons and anti-peripherin antibody (1:100; Millipore) for that of large-diameter DRG axons were used for immunohistochemistry as described previously (Masuda et al., 2003). After staining, the percentage of sections displaying at least one DRG axon had turned in the wrong direction (extending to the intermediate/ventral spinal cord or making a U-turn) was calculated. All data in this study were statistically analyzed by using the Student's $t$ test.

Expression of netrin-1 in human embryonic kidney 293-T cells. Human embryonic kidney (HEK) 293-T cells were transfected with pCAGGS-mouse netrin-1 by using Lipofectamine 2000 reagent (Invitrogen). The cell aggregates were prepared by the hanging drop method as described previously (Kennedy et al., 1994).

Explant cultures. Ventral spinal cords were dissected from E11.5 mouse embryos, and DRGs from E11.5 mouse and stage 24 chick embryos. DRG explants were embedded in a collagen gel $\sim 100-200 \mu \mathrm{m}$ distant from ventral spinal cord explants or aggregates of netrin-1transfected HEK293-T cells. Cultures were performed as described previously (Masuda et al., 2003). For function-blocking studies, an anti-netrin-1 rabbit polyclonal antibody $(2 \mu \mathrm{g} /$ ml; EMD Biosciences) (Watanabe et al., 2006) was added to the culture medium. Collapse assays were performed as described previously (Masuda et al., 2004).

Analysis of DRG axonal growth. For the visualization of DRG axons, cultures were fixed for $1 \mathrm{~d}$ with $4 \%$ PFA. Collagen gels containing tissue explants were then excised and processed for whole-mount immunohistochemistry as described previously (Masuda et al., 2000). After DRG axons had been stained, the length and the trajectory of the axons were traced by using a camera lucida (Nikon). Axons from DRG explants were grouped into four quadrants: proximal, distal, and two lateral ones (supplemental Fig. 2A,C, available at www.jneurosci.org as supplemental material). The length of the 10 longest axons was measured in the proximal and distal quadrants for each culture (Lumsden and Davies, 1983). For evaluation of the effect of netrin-1 on DRG axonal growth, the lengths of the 20 longest axons around DRG explants were measured (Masuda et al., 2000).

In ovo electroporation. In ovo electroporation was performed as described previously (Ono et al., 2004). Briefly, chicken eggs were windowed at stage 16. A mixture of pCAG (cytomegalovirus enhancer-chicken $\beta$-actin promoter)-chick netrin-1 with pGAPgreen fluorescent protein (GFP) $(2 \mu \mathrm{g} / \mu \mathrm{l})$ or pGAP-GFP alone (for control) was injected into the central canal of the spinal cord. Then, electric pulses were delivered to transfect the plasmids into the dorsal spinal cord. The window was sealed with adhesive tape, and the eggs were returned to the incubator for further incubation. Expression of electroporated exogenous netrin- 1 was examined by in situ hybridization. 
Terminal deoxynucleotidyl transferasemediated dUTP-biotin nick end labeling method. For visualization of DNA fragmentation in apoptotic cells, the terminal deoxynucleotidyl transferase-mediated dUTP-biotin nick end labeling (TUNEL) method was performed as described previously (Gavrieli et al., 1992). For double staining with the biotin-16 dUTP and anti-Islet-1/2 (39.4D5; Developmental Studies Hybridoma Bank), streptavidin-Alexa 488 conjugate (Invitrogen), and Alexa Fluor 594 donkey anti-mouse IgG (Invitrogen) were used for detecting the incorporated biotin-16 dUTP by the TUNEL reaction and Islet- $1 / 2$ immunoreactivity, respectively. Islet-1/2- or TUNEL-positive cells were counted and analyzed statistically.

\section{Results}

Expression of netrin-1 and its receptor Unc5c in the mouse embryo

Pioneer DRG axons begin to extend bidirectionally toward their central and peripheral targets at around E10.5 in the mouse embryo (see Fig. 3A) (Ozaki and Snider, 1997). To study the involvement of netrin-1 in these initial DRG axonal projections, we first examined the mRNA expression of netrin-1 in mouse embryos by using a DIG-labeled cRNA probe. Netrin-1 expression was detected in the lateral and medial floor plate and in the dermamyotome at E10.5 (Fig. 1A,B), and its expression continued at E11.5 (Fig. 1C,D).

Recently, in vitro studies provided direct evidence that Unc5 receptors mediate netrin-1-induced repulsion in mammals (Williams et al., 2003; Bartoe et al., 2006). Based on this fact, next we examined Unc5c expression. At the same stages (E10.5-E11.5), Unc5c mRNA was detected in neurons of most parts of DRG as well as in the dermamyotome, epidermis, and ventral horn (Fig. $1 E-H$ ). The expression of the netrin receptor $D C C$, which mediates attraction, was not observed in DRG neurons (data not shown) (Keino-Masu et al., 1996). Unc5c expression in DRG cells continued at E14.5 and was gradually diminished by E17.5 (data not shown). Control sections hybridized with sense probes had no specific signals (data not shown). These expression patterns suggest the possible involvement of netrin-1/Unc5c signaling in the early ventral spinal cord-derived chemorepulsion of DRG axons.

\section{Netrin-1 plays a crucial role in ventral spinal cord-derived chemorepulsion}

To investigate whether netrin-1 acts as an early ventral spinal cord-derived chemorepellent, we cocultured E11.5 mouse DRG explants with mouse netrin-1-transfected cell aggregates for $24 \mathrm{~h}$ in a collagen gel. For quantitative analysis, the length of DRG axons in the proximal quadrant ( $\mathrm{p}$, toward the cells), was compared with that in the distal quadrant ( $\mathrm{d}$, away from the cells). The $\mathrm{p} / \mathrm{d}$ ratio is a measure of repulsive activity, with a ratio of 0 and 1 indicating complete and no repulsion, respectively. Netrin1 -transfected cell aggregates reduced the length of DRG axons in the proximal quadrant compared with that in the distal quadrant (p/d value, 0.52) (Fig. 2A; supplemental Fig. 2A, available at www.jneurosci.org as supplemental material), whereas mocktransfected cell aggregates had no apparent effect (p/d value, 0.964) (Fig. 2B; supplemental Fig. $2 A$, available at www.jneurosci.org as supplemental material). To obtain further evidence for the effect of netrin-1 on early DRG axons, we measured the whole length of DRG axons. Quantitative analyses indicated that netrin-1 reduced DRG axonal length (supplemental Fig. $2 B$, available at www.jneurosci.org as supplemental material) (percentage of DRG axonal length was $74.8 \%$ compared with the control). Thus, these results suggest that netrin-1 both chemorepels and inhibits the outgrowth of early DRG axons. In addition to the axonal growth assay, we performed the collapse assay on DRG growth cones. We observed growth cone collapse of E11.5 mouse DRGs when netrin-1 (500 ng/ml-5 $\mu \mathrm{g} / \mathrm{ml})$ was added to the culture medium (data not shown). These data further indicate that netrin-1 acts on DRG axons directly to restrain their growth.

Based on the above results showing that DRG axons were chemorepelled by netrin-1, we next examined whether netrin-1 participates in early ventral spinal cord-derived chemorepulsion by using netrin-1-deficient tissues. E11.5 mouse DRG explants were 
cocultured in a collagen gel with ventral spinal cord explants from E11.5 wild-type and netrin-1 mutant littermates. DRG neurons extended much shorter axons toward wild-type ventral spinal cord explants, resulting in p/d values of 0.297 (Fig. 2C; supplemental Fig. $2 C$, available at www.jneurosci.org as supplemental material). In contrast, DRG neurons cocultured with netrin-1-deficient ventral spinal cord explants showed significantly increased $\mathrm{p} / \mathrm{d}$ values of 0.618 (Fig. 2 D; supplemental Fig. $2 C$, available at www.jneurosci.org as supplemental material). Together with the netrin-1 expression pattern shown above, these results suggest that the early ventral spinal cord utilizes netrin-1 to chemorepel DRG axons.

To further define the role of netrin-1 in early ventral spinal cord-derived chemorepulsion, we used a function-blocking antibody against mouse netrin-1 (2 $\mu \mathrm{g} / \mathrm{ml}$; Watanabe et al., 2006) to perturb the effect of netrin-1 in cocultures. We cocultured E11.5 mouse ventral spinal cord explants with E11.5 mouse or stage 24 chick DRGs [stage 24 is the earliest stage for culturing chick DRGs to examine their axonal response to guidance cues (Nakamoto and Shiga, 1998)]. The addition of the netrin-1 antibody significantly increased the $\mathrm{p} / \mathrm{d}$ values ( 0.524 for mouse, 0.57 for chick) (Fig. $2 E, F$; supplemental Fig. $2 D$, available at www.jneurosci.org as supplemental material). These results further indicate that netrin- 1 is involved in chemorepulsive signals from the ventral spinal cord in a speciesindependent manner in higher vertebrates.

\section{Defects in axonal trajectories at initial stages of DRG axonal projection in netrin-1 mutant mice}

Next, we examined DRG axonal trajectories in vivo in netrin-1 mutant mice at E10 and E13.5. In E10 netrin-1 mutants, we observed that a subset of axons from the DRG did not project to the dorsal root entry zone (DREZ) but rather projected aberrantly toward the intermediate and ventral regions of the spinal cord (Fig. $3 B, D, F)$ [ 10 sections of the 34 sections examined showed aberrant projections toward the intermediate or ventral spinal cord $(29.4 \%)$, whereas only 1 section showed the misorientation toward the dorsal lip of the dermamyotome $(2.9 \%) ; n=3$ ]. In contrast, we found that most DRG axons in wild-type embryos projected normally toward the DREZ (Fig. $3 A, C, E)(8 \%$ of the 50 sections examined showed aberrant projections; $n=4 ; p<0.01)$. These results strongly suggest that netrin-1 is necessary for the correct projection of DRG axons.

To determine whether the misorientation of DRG axons in netrin-1 mutants was caused by the malformation of DRG neurons, we examined the number of apoptotic and ectopic DRG neurons by using the TUNEL method to detect cell death and immunostaining for Islet-1/2 to detect the location of DRG neurons (Cui and Goldstein, 2000). No significant difference in either apoptosis or the position of DRGs was observed between E10 wild-type (213 sections; $n=3$ ) (supplemental Fig. 3, available at www.jneurosci.org as supplemental material) (number of TUNEL-positive cells $=1.11$ per section, number of ectopic cells $=0.43$ per section) and netrin- 1 mutant littermates (152 sections; $n=3$ ) (supplemental Fig. 3, available at www. jneurosci.org as supplemental material) [number of TUNELpositive cells $=1.12$ per section $(p=0.969)$, number of ectopic cells $=0.37$ per section $(p=0.623)]$. Therefore, DRG neurons are normally formed in the absence of netrin-1 signaling.

We also examined wild-type $(n=4)$ and netrin- 1 mutant $(n=$ 4) mice at later stages (E13.5) and found no aberrant DRG axonal projection in relation to the ventral spinal cord in either genotype (Fig. $3 G, H$ ). These results suggest that the aberrant projection of DRG axons in E10 netrin-1 mutants is transient and subsequently eliminated.
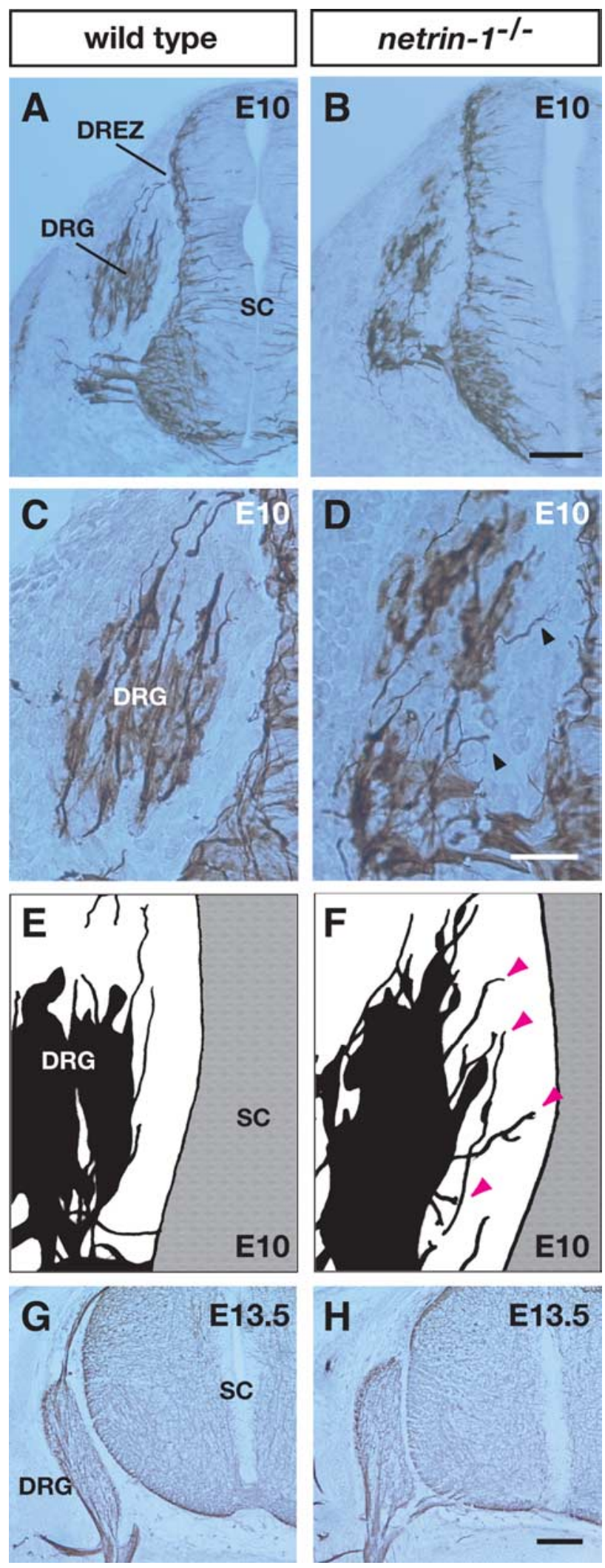

Figure 3. Defects in axonal trajectories at initial stages of DRG axonal projection in netrin- $1 \mathrm{mu}-$ tants. $A, B$, Transverse sections of $E 10$ wild-type $(\boldsymbol{A})$ and netrin- 1 mutant $(\boldsymbol{B})$ littermates labeled with an anti-class III $\beta$-tubulin antibody. $\boldsymbol{C}, \boldsymbol{D}$, Higher-magnification views of the DRG from $A$ and $\boldsymbol{B}$, respectively. Misrouted DRG axons are observed in netrin- 1 mutant littermates (arrowheads). $\boldsymbol{E}, \boldsymbol{F}$, Camera lucida drawings showing axons and cell bodies of DRGs in sections of wild-type $(\boldsymbol{E})$ and netrin-1 mutant $(\boldsymbol{F})$ littermates. Arrowheads (magenta) indicate misrouted axons in the netrin- 1 mutant. $\mathbf{G}, \boldsymbol{H}$, In contrast to E10, there are no aberrant DRG axonal projections in sections of E13.5 wild-type $(\boldsymbol{G})$ or netrin-1 mutant $(\boldsymbol{H})$ littermates. SC, Spinal cord. Scale bars: $\boldsymbol{B}, \boldsymbol{H}, 100 \mu \mathrm{m} ; \boldsymbol{D}, 50 \mu \mathrm{m}$. 


\section{Misexpression of netrin- 1 by the spinal cord perturbs DRG axonal projection toward the DREZ}

To test whether netrin-1 regulates the in vivo behavior of DRG axons directly, we introduced the netrin-1 expression vector into the chick spinal cord by electroporation. Because chick DRG cells extend axons toward their target at stage 18-24 (Shiga et al., 2000; our unpublished observations), the netrin-1expressing vector was electroporated into stage 16 chick spinal cords, and trajectories of DRG axons were examined at stage 22. In the three control embryos that received GFP alone, DRG axons normally projected toward the DREZ (Fig. $4 A, B)(1.8 \%$ of the 113 sections examined showed aberrant projections; number of axons reaching the DREZ = 2.78 per section in the control embryos). In contrast, in the four embryos that misexpressed netrin- 1 in their dorsal and intermediate spinal cord, we observed that a subset of DRG axons failed to orient toward the DREZ and turned in the wrong direction; i.e., the aberrant branches or axons of DRG neurons extended to the dermamyotome (Fig. $4 C, D$ ) or made a U-turn (Fig. $4 E, F$ ) [29.1\% of the 148 sections examined showed aberrant projections $(p<0.0001)$; the number of axons reaching the DREZ $=1.79$ per section $(p<0.05)$ in the netrin-1misexpressing embryos]. Our results thus provide in vivo evidence that the misexpression of netrin- 1 in the dorsal spinal cord controls and alters the direction of DRG axons.

\section{Discussion}

Chemorepulsive guidance cues derived from surrounding nontarget tissues play an important role in the guidance of DRG axons toward the dorsal spinal cord (Keynes et al., 1997; Nakamoto and Shiga, 1998). However, the identity of molecules involved in the ventral spinal cord-derived chemorepulsion is not well understood. Early DRG axons are sandwiched between the dermamyotome and the ventral spinal cord, both of which express netrin-1, and DRG neurons express the netrin receptor Unc5c. This expression pattern suggests that early DRG neurons respond to netrin-1-induced chemorepulsion. In fact, our coculture study using netrin-1-expressing cells shows that netrin-1 has a chemorepulsive activity toward DRG axons. Furthermore, the chemorepulsive activity of netrin-1-deficient ventral spinal cord explants is much weaker than that of the wild-type ones. We observe that some DRG axons in netrin-1 mutants do not project toward the DREZ in vivo but proceed to the intermediate or ventral spinal cord. In contrast, we also observe that some DRG axons fail to reach the DREZ but proceed to the dermamyotome when netrin-1 was misexpressed in the spinal cord in vivo. Together, both our in vivo and in vitro results strongly suggest that netrin-1 derived from the ventral spinal cord acts as a chemorepellent for DRG axons.

\section{Early ventral spinal cord-derived repulsion by netrin-1}

Our highly sensitive in situ hybridization technique detects a broad expression pattern of netrin-1 mRNA in the entire floor plate. Consistent with this finding, a previous study using an antibody against netrin-1 revealed a broad gradient of netrin-1 protein throughout the spinal cord at early stages (Kennedy et al., 2006). Although netrin-1 protein expression in the mesenchymal region appears to be very weak in that study, the netrin-1 mutant phenotype showing DRG axonal elongation toward the intermediate and ventral spinal cord strongly indicates that the secretion of netrin-1 into the mesenchyme surrounding the spinal cord plays a guidance role for DRG axons. Moreover, our finding that the misexpression of netrin-1 in the dorsal spinal cord causes pathfinding errors of DRG axons further strengthens the idea that the long-range gradient of netrin-1 protein plays important roles in DRG axonal guidance at early stages.

Netrin-1 is expressed in the floor plate from early to late stages of DRG development (Wang et al., 1999). Because netrin-1 mutants exhibit correct ventral projections by large-diameter DRG axons (NT-3-dependent proprioceptive axons) despite a severe disorganization of the dorsal funiculus at E15.5 (supplemental Fig. 4, available at www.jneurosci.org as supplemental material), netrin-1 in the ventral spinal cord may not act as a repellent at later stages. In fact, the expression of the netrin receptor Unc 5 c is downregulated in the DRG of late stage embryos (Guan and Condic, 2003; our unpublished observation). Because of the downregulation of Unc5c, large-diameter DRG axons are not repelled by ventrally derived netrin- 1 and therefore can thus project from the dorsal funiculus toward the motor pool in the ventral spinal cord. Thus, netrin-1/Unc5c signaling may be involved in early but not later ventral spinal cord-derived chemorepulsion.

\section{Molecular mechanisms mediating ventral spinal cord-derived repulsion}

We find that netrin-1-deficient ventral spinal cords retain weak repulsive activities and that the anti-netrin-1 antibody does not completely eliminate the ventral spinal cord-derived repulsion. These results strongly suggest the possibility that the ventral spi- 
nal cord utilizes repulsive signals in addition to netrin-1/Unc5c. In fact, $U n c 5 c$ mRNA is not detected in all DRG neurons. The absence of $U n c 5 c$ expression in the subpopulation of DRG neurons may reflect the existence of unaffected DRG axons despite loss- or gain-of-function manipulations of netrin-1. We previously showed that a member of the IgFnIII CAM family, TAG-1/ axonin-1/SC2, which is expressed on DRG axons, participates in mediating early ventral spinal cord-derived repulsion (Masuda et al., 2003; our unpublished observations). It is possible that TAG1/axonin-1/SC2 might mediate ventral spinal cord-derived chemorepulsion other than netrin-1. Alternatively, TAG-1/axonin1/SC2 may interact with Unc5c to mediate the chemorepulsive activity of netrin-1. Further studies will determine whether TAG1/axonin-1/SC2 collaborates with Unc5c to mediate netrin-1 signaling.

In conclusion, we provide evidence that netrin-1 plays a key role in early DRG axonal guidance and contributes to the initial formation of DRG axonal projections to the dorsal spinal cord.

\section{References}

Bartoe JL, McKenna WL, Quan TK, Stafford BK, Moore JA, Xia J, Takamiya K, Huganir RL, Hinck L (2006) Protein interacting with C-kinase 1/protein kinase $\mathrm{C} \alpha$-mediated endocytosis converts netrin-1-mediated repulsion to attraction. J Neurosci 26:3192-3205.

Colamarino SA, Tessier-Lavigne M (1995) The axonal chemoattractant netrin-1 is also a chemorepellent for trochlear motor axons. Cell 81:621-629.

Cui S, Goldstein RS (2000) Early markers of neuronal differentiation in DRG: islet-1 expression precedes that of Hu. Brain Res Dev Brain Res 121:209-212.

Ding L, Takebayashi H, Watanabe K, Ohtsuki T, Tanaka KF, Nabeshima Y, Chisaka O, Ikenaka K, Ono K (2005) Short-term lineage analysis of dorsally derived Olig3 cells in the developing spinal cord. Dev Dyn 234:622-632.

Gavrieli Y, Sherman Y, Ben-Sasson SA (1992) Identification of programmed cell death in situ via specific labeling of nuclear DNA fragmentation. J Cell Biol 119:493-501.

Guan W, Condic ML (2003) Characterization of Netrin-1, Neogenin and cUNC-5H3 expression during chick dorsal root ganglia development. Gene Exp Pat 3:369-373.

Hamburger V, Hamilton HL (1992) A series of normal stages in the development of the chick embryo. Dev Dyn 195:231-272.

Hong K, Hinck L, Nishiyama M, Poo MM, Tessier-Lavigne M, Stein E (1999) A ligand-gated association between cytoplasmic domains of UNC5 and DCC family receptors converts netrin-induced growth cone attraction to repulsion. Cell 97:927-941.

Keino-Masu K, Masu M, Hinck L, Leonardo ED, Chan SS, Culotti JG, TessierLavigne M (1996) Deleted in Colorectal Cancer (DCC) encodes a netrin receptor. Cell 87:175-185.

Kennedy TE, Serafini T, de la Torre JR, Tessier-Lavigne M (1994) Netrins are diffusible chemotropic factors for commissural axons in the embryonic spinal cord. Cell 78:425-435.

Kennedy TE, Wang H, Marshall W, Tessier-Lavigne M (2006) Axon guid- ance by diffusible chemoattractants: a gradient of netrin protein in the developing spinal cord. J Neurosci 26:8866-8874.

Keynes R, Tannahill D, Morgenstern DA, Johnson AR, Cook GM, Pini A (1997) Surround repulsion of spinal sensory axons in higher vertebrate embryos. Neuron 18:889-897.

Lumsden AG, Davies AM (1983) Earliest sensory nerve fibres are guided to peripheral targets by attractants other than nerve growth factor. Nature 306:786-788.

Masuda T, Okado N, Shiga T (2000) The involvement of axonin-1/SC2 in mediating notochord-derived chemorepulsive activities for dorsal root ganglion neurites. Dev Biol 224:112-121.

Masuda T, Tsuji H, Taniguchi M, Yagi T, Tessier-Lavigne M, Fujisawa H, Okado N, Shiga T (2003) Differential non-target-derived repulsive signals play a critical role in shaping initial axonal growth of dorsal root ganglion neurons. Dev Biol 254:289-302.

Masuda T, Fukamauchi F, Takeda Y, Fujisawa H, Watanabe K, Okado N, Shiga T (2004) Developmental regulation of notochord-derived repulsion for dorsal root ganglion axons. Mol Cell Neurosci 25:217-227.

Nakamoto K, Shiga T (1998) Tissues exhibiting inhibitory and repulsive activities during the initial stages of neurite outgrowth from the dorsal root ganglion in the chick embryo. Dev Biol 202:304-314.

Ono K, Yasui Y, Ikenaka K (2004) Lower rhombic lip-derived cells undergo transmedian tangential migration followed by radial migration in the chick embryo brainstem. Eur J Neurosci 20:914-922.

Ozaki S, Snider WD (1997) Initial trajectories of sensory axons toward laminar targets in the developing mouse spinal cord. J Comp Neurol 380:215-229.

Serafini T, Kennedy TE, Galko MJ, Mirzayan C, Jessell TM, Tessier-Lavigne M (1994) The netrins define a family of axon outgrowth-promoting proteins homologous to C. elegans UNC-6. Cell 78:409-424.

Serafini T, Colamarino SA, Leonardo ED, Wang H, Beddington R, Skarnes WC, Tessier-Lavigne M (1996) Netrin-1 is required for commissural axon guidance in the developing vertebrate nervous system. Cell 87:1001-1014.

Shiga T, Kawamoto M, Shirai T (2000) Longitudinal elongation of primary afferent axons in the dorsal funiculus of the chick embryo spinal cord. Dev Brain Res 124:25-31.

Sugimoto Y, Taniguchi M, Yagi T, Akagi Y, Nojyo Y, Tamamaki N (2001) Guidance of glial precursor cell migration by secreted cues in the developing optic nerve. Development 128:3321-3330.

Taniguchi M, Yuasa S, Fujisawa H, Naruse I, Saga S, Mishina M, Yagi T (1997) Disruption of semaphorin III/D gene causes severe abnormality in peripheral nerve projection. Neuron 19:519-530.

Tessier-Lavigne M, Goodman CS (1996) The molecular biology of axon guidance. Science 274:1123-1133.

Wang H, Copeland NG, Gilbert DJ, Jenkins NA, Tessier-Lavigne M (1999) Netrin-3, a mouse homolog of human NTN2L, is highly expressed in sensory ganglia and shows differential binding to netrin receptors. J Neurosci 19:4938-4947.

Watanabe K, Tamamaki N, Furuta T, Ackerman SL, Ikenaka K, Ono K (2006) Dorsally derived netrin 1 provides an inhibitory cue and elaborates the "waiting period" for primary sensory axons in the developing spinal cord. Development 133:1379-1387.

Williams ME, Wu SC, McKenna WL, Hinck L (2003) Surface expression of the netrin receptor $\mathrm{UNC5H} 1$ is regulated through a protein kinase C-interacting protein/protein kinase-dependent mechanism. J Neurosci 23:11279-11288. 